\title{
Synthesis and Characterization of New 8-trifluloromethyl Quinazolin-2,4-(3H)-Dione Nucleosides
}

\author{
Laila M. Break \\ Correspondence: Laila M. Break, Taif University, Al-Haweiah, P.O.Box 888 Zip Code 21974. Taif, Saudi Arabia. \\ E-mail: lailabreek@gmail.com
}

Received: January 3, 2017 Accepted: January 20, 2017 Online Published: January 23, 2017

doi:10.5539/ijc.v9n1p73

URL: http://dx.doi.org/10.5539/ijc.v9n1p73

\begin{abstract}
Synthesis of 8-trifluloromethyl quinazolin-2,4- $(1 H, 3 H)$-dione 2 . which have been ribosylated by coupling with 1- $O$-acetyl-2,3,5-tri- $O$-benzoyl- $\beta$-D-ribofuranose 4 by using the silylation method, afforded mixture $\beta$-and $\alpha$-anomeric of the benzoylated nucleoside derivatives 5 and $\mathbf{6}$, respectively. Debenzoylation of each of 5 and $\mathbf{6}$ by sodium metal in dry methanol to afford the corresponding free nucleosides $\mathbf{7}$ and $\mathbf{8}$ respectively. The structures of the newly synthesis compounds have been confirmed on the basis of elemental analyses, IR, ${ }^{1} \mathrm{HNMR},{ }^{13} \mathrm{CNMR}$ and Mass spectral data.
\end{abstract}

Keywords:1-O-Acetyl-2,3,5-trihydroxy- $\beta$-D-ribofuranose, $\quad$ Nucleosides, $\quad$ Quinazolin-2,4-(1H,3H)-dione, Trifluoromethyl

\section{Introduction}

Quinazolinone is a heterocyclic compound that occupies a distinct and place in the field of medicinal chemistry. Many of them were showed antimicrobial, anti-inflammatory, anticonvulsant, analgesic and anticancer agents (Safinaz E.Abbas et al, 2013; A. Kumar et al, 2011; K.M. Amin et al, 2010; M.M. Aly, 2010; A. Kumar, 2003; S.T. Al-Rashood, 2006 and N. Mulakayala, 2012).

Quinazoline nucleosides were first synthesized by Stout and Robins in 1968 as pyrimidine nucleoside analogs (Stout M. G and Robins R. K., 1968) and consequent synthetic studies were contributed by Dunkel and Pfleiderer in the 1990s. (Dunkel M and Pfleiderer W, 1991, 1992 and1993). More recently, several quinazoline-2,4-dione nucleosides have been incorporated into oligonucleotides as pyrimidine nucleoside substitutes to study the binding affinity and base pairing selectivity(Michel J et al, 1997; Diwan A. R., 1969; T. C. Chien, 2005; F. E. M. El-Baih, 2004; Tun-Cheng CHIEN, 2004).

Many familiar drugs and pharmacological studies contain trifluoromethyl groups. Quinazoline-2,4-diones bearing a trifluoromethyl group derivatives were an inhibitor of human immunodeficiency virus-1 reverse transcriptase, antagonists at ionotropic glutamate receptors (Hao Chen et al, 2003; Vittoria Colotta, 2012) and anticancer compound trifluoromethyl-substituted pyrazole N-nucleoside(Ayman M. Saleh et al, 2016).

In this review, quinazolin-2,4-(3H)-dione nucleosides containing trifluloromethyl group were designed as part of our continuing interest in the synthesis of new nucleosides as expected their biological activity.

\section{Material and Methods}

Melting points were measured on Gallenkamp melting point apparatus (UK) and are uncorrected. The purity of the compounds was checked by thin layer chromatography (TLC). Thin layer chromatography (TLC) was performed on silica gel sheets F1550 LS 254 of Schleicher \& Schull and column chromatography on Merck silica gel 60 (particle size 0.063-0.20. Elemental analyses were obtained on an Elementary Vario EL 1150C analyzer. IR spectra were recorded on $\mathrm{KBr}$ discs on Fourier Transform infrared and Pie Unicom SP 300 Infrared Spectrophotometers at Taif University. ${ }^{1} \mathrm{H}$ NMR and ${ }^{13} \mathrm{C}$ NMR spectra were obtained on a Varian $(850 \mathrm{MHz})$ EM 390 USA instrument at King Abdel-Aziz University by using TMS as the internal reference. Mass spectra were recorded on a JEOL-JMS-AX500 at King Abdel-Aziz University, Saudi Arabia.

\section{Experimental}

\section{8-trifluloromethyl quinazolin-2,4-(1H,3H)-dione 2}

2-Amino-3-trifluloromethyl benzoic acid 1 (Aldrich; 0.019mol, 4g) was added drops of acetic acid in (100 ml) water, 
the solution of $\mathrm{KNCO}(0.049 \mathrm{~mol}, 4 \mathrm{~g})$ was dropped to the mixture was stirred in an ice bath at $1 \mathrm{~h}$. The reaction mixture was added sodium hydroxide $(10 \mathrm{~g})$ and overnight at room temperature and then filtered. The precipitate was neutralized with dilute sulfuric acid $(1: 1)$ and washed $(3 \times 20 \mathrm{ml})$ water. The compound purified by column chromatography on silica gel with (Ethylacetate: Acetone 9:1) to afford white crystals.

Yield (63.23\%), w. 6.1g, m.p. $<300^{\circ} \mathrm{C}$ white ; $v\left(\mathrm{~cm}^{-1}\right)(\mathrm{KBr}) 3400,3075,1740,1640 ;{ }^{1} \mathrm{HNMR}$ (850MHz); (DMSO-D ${ }_{6}$ ): $11.62(\mathrm{~s}, 1 \mathrm{H}) \mathrm{NH}-1,11.20(\mathrm{~s}, 1 \mathrm{H}) \mathrm{NH}-3, \quad 8.22(\mathrm{~s}, 1 \mathrm{H}) \mathrm{H}_{5}, 7.79(\mathrm{~d}, 1 \mathrm{H}, J=7.5 \quad \mathrm{~Hz}) \mathrm{H}_{7}, 7.18(\mathrm{~d}, 1 \mathrm{H}, J=7.5 \mathrm{~Hz}) \mathrm{H}_{6}$. ${ }^{13} \mathrm{CNMR}(850 \mathrm{MHz}): \delta 167.27,154.77,133.08,130.82,128.39,125.71,124.15,123.09,109.80 ; \quad \mathrm{MS} \mathrm{m} / \mathrm{z}: 230\left(\mathrm{M}^{+}\right.$, 45\%). Anal. Calcd. for $\mathrm{C}_{9} \mathrm{H}_{5} \mathrm{~F}_{3} \mathrm{~N}_{2} \mathrm{O}_{2}$; M.wt: 230.14; C,46.97; H,2.19; F,24.77; N, 12.17 (\%); Found: C, 46.26; H, 2.89; $\mathrm{F}, 24.01 ; \mathrm{N}, 11.05(\%)$.

Synthesis of protection nucleoside of 8-trifluloromethyl quinazolin-2,4-(1H,3H)-dione 2

\section{General Procedure}

Silyation of 8-trifluloromethyl quinazolin-2,4-( $1 \mathrm{H}, 3 \mathrm{H})$-dione 2 ( $0.021 \mathrm{~mol})$ with hexamethyldisilazane (HMDS) (20 ml) was refluxed for 3 days with a catalytic a few crystals of ammonium sulfate under exclusion of moisture. Excess of HMDS was removed in vacuo by co-evaporation with dry dichloroethane gave the silyated derivative $\mathbf{3}$, using the Vorbruggen's silylation method (Vorbruggen et al, 1981). The residue was dissolved in $20 \mathrm{ml}$ of dry 1,2-dichloroethane and then 1- $O$-acetyl-2,3,5-tri- $O$-benzoyl- $\beta$-D-ribofuranose $4(10.8 \mathrm{~g}, 0.021 \mathrm{~mol})$ was added. The mixture was added dropwise onto a mixture $(4.5 \mathrm{ml})$ of $(10 \mathrm{ml}$ trimethylsilyl trifluoromethane sulfonate (TMSOTf) in dry 1,2-dichloroethane $(50 \mathrm{ml})$ ). The mixture was stirred at room temperature for $24 \mathrm{~h}$, and then washed with a saturated solution of aqueous sodium bicarbonate $(3 \times 50 \mathrm{ml})$, washed with water $(3 \times 50 \mathrm{ml})$, and dried over anhydrous sodium sulfate. the organic phase was extracted by $\mathrm{CH}_{2} \mathrm{Cl}_{2}$, dried over $\mathrm{MgSO}_{4}$ and evaporated. The solvent was removed under vacuum gave an anomeric mixture of $\beta$ and $\alpha$-1-(2,3,5-tri- $O$-benzoyl-D-ribofuranosyl)8-trifluloromethylquinazolin-2,4-(3H)-dione. The protected nucleoside was separated by column chromatography on silica gel with dichloromethane: acetone (9:1) as eluent to afford a white crystal pure $\beta$-anomeric 5 and $\alpha$-anomeric $\mathbf{6}$ respectively, in good yields.

\section{$\boldsymbol{\beta}$-1-(2,3,5-Tri- $O$-benzoyl-D-ribofuranosyl)-8-trifluloromethyl quinazolin-2,4-(3H)-dione 5}

Yield (52.12\%), w. 2.7 g, m.p. $119^{\circ} \mathrm{C}$ white; IR $v\left(\mathrm{~cm}^{-1}\right)(\mathrm{KBr}) 3042,1725,1680 ;{ }^{1} \mathrm{HNMR}(850 \mathrm{MHz}) ;\left(\mathrm{CDCl}_{3}\right): \delta 9.47(\mathrm{~s}$, $1 \mathrm{H}) \mathrm{H}_{3 \text { Amide }}, 8.26(\mathrm{~d}, 1 \mathrm{H}, J=7.5 \mathrm{~Hz}) \mathrm{H}_{5}, 8.03(\mathrm{~d}, 1 \mathrm{H}, J=7.2 \mathrm{~Hz}) \mathrm{H}_{7}, 7.92(\mathrm{dd}, 1 \mathrm{H}, J=15.7 \mathrm{~Hz}) \mathrm{H}_{6}, 7.51-7.25(\mathrm{~m}, 15 \mathrm{H})$ $\mathrm{H}_{(\mathrm{Ar}-\mathrm{H})}, 6.48(\mathrm{~d}, 1 \mathrm{H}, J=7.5 \mathrm{~Hz}) \mathrm{H}_{1^{\prime}}, 6.16(\mathrm{dd}, 1 \mathrm{H}, J=8.4 \mathrm{~Hz}) \mathrm{H}_{2}, 6.09(\mathrm{t}, 1 \mathrm{H}, J=13.4 \mathrm{~Hz}) \mathrm{H}_{3}, 4.82-4.77(\mathrm{dd}, 1 \mathrm{H}, J=$ $4.6 \mathrm{~Hz}) \mathrm{H}_{5}, 4.69-4.56(\mathrm{~m}, 1 \mathrm{H}) \mathrm{H}_{4} \cdot{ }^{13} \mathrm{CNMR}(850 \mathrm{MHz})\left(\mathrm{CDCl}_{3}\right): \delta 166.30,165.46,164.87,165.53_{\mathrm{C}=\mathrm{O} \text { 's groups }}, 148.17 \mathrm{C}_{4}$, 146.78 $\mathrm{C}_{2}, 133.61-128.33$ Ar-carbons, $119.11 \mathrm{CF}_{3}, 88.02 \mathrm{C}_{1}, 79.47 \mathrm{C}_{2}, 73.93 \mathrm{C}_{3}, 70.98 \mathrm{C}_{4}, 63.82 \mathrm{C}_{5}$ sugar carbons. Anal. Calcd. for $\mathrm{C}_{35} \mathrm{H}_{25} \mathrm{~F}_{3} \mathrm{~N}_{2} \mathrm{O}_{9}$; M.wt: 674.58; C,62.32; H,3.74; F,8.45; N, 4.15; (\%); Found: C, 62.26; H, 3.89; F,8.21; N,3.95 (\%).

\section{$\alpha$-1-(2,3,5-Tri- $O$-benzoyl-D-ribofuranosyl)-8-trifluloromethyl quinazolin-2,4-(3H)-dione 6}

Yield (47.87\%), w. 2.48 g, m.p. $105-107^{\circ} \mathrm{C}$ white color; IR $v\left(\mathrm{~cm}^{-1}\right)(\mathrm{KBr}) 3020,1725,1685 ;{ }^{1} \mathrm{HNMR}(850 \mathrm{MHz})$ $\left(\mathrm{CDCl}_{3}\right): \delta 9.16(\mathrm{~s}, 1 \mathrm{H}) \mathrm{H}_{\text {Amide }}, 8.06(\mathrm{~d}, 1 \mathrm{H}, J=7.4 \mathrm{~Hz}) \mathrm{H}_{5}, 7.98(\mathrm{~d}, 1 \mathrm{H}, J=7.2 \mathrm{~Hz}) \mathrm{H}_{7}, 7.89(\mathrm{~d}, 1 \mathrm{H}, J=7.4 \mathrm{~Hz}) \mathrm{H}_{6}$, 7.55-7.25 (m, $15 \mathrm{H}) \mathrm{H}_{(\mathrm{Ar}-\mathrm{H})}, 6.73(\mathrm{~d}, 1 \mathrm{H}, J=2.2 \mathrm{~Hz}) \mathrm{H}_{1}, 6.14(\mathrm{dd}, 1 \mathrm{H}, J=8.8 \mathrm{~Hz}) \mathrm{H}_{2}, 6.03-5.89(\mathrm{~m}, 1 \mathrm{H}) \mathrm{H}_{3}, 4.81-4.57$ $(\mathrm{m}, 1 \mathrm{H}) \mathrm{H}_{4}, \quad 3.73(\mathrm{~m}, 1 \mathrm{H}) \mathrm{H}_{5} \cdot{ }^{13} \mathrm{CNMR}(850 \mathrm{MHz}): \delta 166.46,165.82,165.40{ }_{\mathrm{C}=\mathrm{O}^{\prime} \text { groups }}, 147.95 \mathrm{C}_{4}, 147.26 \mathrm{C}_{2}$, 13.73-128.40 Aromatic carbons, $118.01 \quad \mathrm{CF}_{3}, 87.46 \mathrm{C}_{1}, 79.37 \mathrm{C}_{2}, 73.90 \mathrm{C}_{3}, 71.69 \mathrm{C}_{4}, \quad 63.83 \mathrm{C}_{5} . \quad\left(\mathrm{CH}_{3} \mathrm{Cl}\right.$ : $\mathrm{CH}_{3} \mathrm{COOCH}_{2} \mathrm{CH}_{3}$ ) (9:1). Anal. Calcd. for $\mathrm{C}_{35} \mathrm{H}_{25} \mathrm{~F}_{3} \mathrm{~N}_{2} \mathrm{O}_{9}$; M.wt: 674.58; C,62.32; H,3.74; F,8.45; N, 4.15; (\%); Found: C, $62.15 ; \mathrm{H}, 3.45 ; \mathrm{F}, 8.91 ; \mathrm{N}, 4.09(\%)$.

Deprotection of $\mathbf{5}$ and $\mathbf{6}$. Synthesis of free nucleosides $\mathbf{7}$ and $\mathbf{8}$ respectively

\section{General Procedure}

The pure anomer of each $\beta \mathbf{5}$ and $\alpha \mathbf{6}(0.001 \mathrm{~mol}$ for each), dry absolute methanol $(20 \mathrm{ml})$ and sodium metal $(0.055 \mathrm{~g}$, $0.001 \mathrm{~mol}$ ) was stirred at room temperature for $48 \mathrm{~h}$. The solvent was evaporated under vacuum to give a colorless solid, which was dissolved in hot water and neutralized with few drops acetic acid. Purification of each compound by TLC chromatographic on silica gel with chloroform: ethyl acetate (9: 1) to afford colorless and white crystals of the following Zemplen et al.'s method (Zemplen et al, 1939) to afford the free nucleosides $\mathbf{7}$ and $\mathbf{8}$, respectively.

\section{$\boldsymbol{\beta}$-1-(2,3,5-Trihydroxy-D-ribofuranosyl)-8-trifluloromethyl quinazolin-2,4-(3H)-dione 7}

Yield (81.67\%), w. 0.307g. m.p. $185^{\circ} \mathrm{C}$ white color; IR $v\left(\mathrm{~cm}^{-1}\right)(\mathrm{KBr}) 3450,3032,1715,1685 ;{ }^{1} \mathrm{HNMR}$ (600MHz)(DMSO-D $\left.)_{6}\right): \delta 11.59(\mathrm{~s}, 1 \mathrm{H}) \quad \mathrm{H}_{1 \text { Amide, }} 8.06(\mathrm{~d}, 1 \mathrm{H}, J=5.5 \mathrm{~Hz}) \mathrm{H}_{5}, 7.80-7.79(\mathrm{~d}, 1 \mathrm{H}, J=8.7 \mathrm{~Hz}) \mathrm{H}_{7}$, 7.77-7.76 (d, $1 \mathrm{H}, J=5.2 \mathrm{~Hz}) \mathrm{H}_{6}, 6.17(\mathrm{~d}, 1 \mathrm{H}, J=7.5 \mathrm{~Hz}) \mathrm{H}_{1}, 5.27(\mathrm{~s}, 1 \mathrm{H}) \mathrm{H}_{2}, 5.07(\mathrm{~m}, 1 \mathrm{H}) \mathrm{H}_{3}, 4.45(\mathrm{t}, 1 \mathrm{H}) \mathrm{H}_{5}, 4.13$ (s, $1 \mathrm{H}) \mathrm{H}_{4}, 3.80-3.76(\mathrm{~m}, 1 \mathrm{H}) \mathrm{H}_{2 \cdot \mathrm{OH}}, 3.66-3.61(\mathrm{~m}, 1 \mathrm{H}) \mathrm{H}_{3^{\circ} \mathrm{OH}}, 3.58-3.41(\mathrm{~m}, 1 \mathrm{H}) \mathrm{H}_{3 \cdot \mathrm{OH}} \cdot{ }^{13} \mathrm{C} \mathrm{NMR}: \quad 155.07 \mathrm{C}_{4}, 153.63$ $\mathrm{C}_{2}, 139.6,137.59,133.73,129.92,128.86,120.00,118.96 \mathrm{CF}_{3}, 89.89 \mathrm{C}_{1}, 85.31 \mathrm{C}_{2}, 69.41 \mathrm{C}_{3}, 69.29 \mathrm{C}_{4}, 61.63 \mathrm{C}_{5}$. 
$\left(\mathrm{CH}_{3} \mathrm{COOCH}_{2} \mathrm{CH}_{3}\right.$ : Acetone) (9:1); $\mathrm{MS} \mathrm{m} / \mathrm{z}: 362\left(\mathrm{M}^{+}, 21 \%\right)$. Anal. Calcd. for $\mathrm{C}_{14} \mathrm{H}_{13} \mathrm{~F}_{3} \mathrm{~N}_{2} \mathrm{O}_{6} ;$ M.wt: 362.26; C,46.42; H,3.62; F,15.73; N, 7.73; (\%); Found: C, 46.12; H, 3.75; F,15.91; N,7.24 (\%).

\section{$\alpha$-1-(2,3,5-Trihydroxy-D-ribofuranosyl)-8-trifluloromethyl quinazolin-2,4-(3H)-dione 8}

Yield $(94.73 \%)$, w. 0.41 g, m.p. $220^{\circ} \mathrm{C}$ white color; IR $v\left(\mathrm{~cm}^{-1}\right)(\mathrm{KBr}) 3450,1714,1690 ;{ }^{1} \mathrm{HNMR}$ $(600 \mathrm{MHz})\left(\right.$ DMSO-D $\left._{6}\right): \delta 11.85(\mathrm{~s}, 1 \mathrm{H}) \mathrm{H}_{1 \text { Amide }}, 8.06(\mathrm{~d}, 1 \mathrm{H}, J=1.46 \mathrm{~Hz}) \mathrm{H}_{5}, 7.80(\mathrm{t}, 1 \mathrm{H}, J=11.37 \mathrm{~Hz}) \mathrm{H}_{7}, 7.77(\mathrm{~d}, 1 \mathrm{H}$, $J=1.83 \mathrm{~Hz}) \mathrm{H}_{8}, 6.53(\mathrm{~d}, 1 \mathrm{H}, J=4.5 \mathrm{~Hz}) \mathrm{H}_{1}, 5.25(\mathrm{~d}, 1 \mathrm{H}, J=5.87 \mathrm{~Hz}) \mathrm{H}_{2}, 5.05(\mathrm{t}, 1 \mathrm{H}, J=21.65 \mathrm{~Hz}) \mathrm{H}_{3}, 4.46(\mathrm{q}, 1 \mathrm{H})$ $\mathrm{H}_{5}, 4.13(\mathrm{q}, 1 \mathrm{H}) \mathrm{H}_{4}, 3.8(\mathrm{~m}, 2 \mathrm{H}) \mathrm{H}_{4 \mathrm{a}}, 3.60(\mathrm{~s}, 1 \mathrm{H}) \mathrm{OH}_{2}, 3.58(\mathrm{~s}, 1 \mathrm{H}) \mathrm{OH}_{3,3.57}(\mathrm{~s}, 1 \mathrm{H}) \mathrm{OH}_{5} \cdot{ }^{13} \mathrm{C}_{\mathrm{NMR}}$ : $161.07 \mathrm{C}_{4}$, $150.63 \mathrm{C}_{2}, 139.67,137.59,133.73,129.93,125.34,120.00,118.82 \mathrm{CF}_{3}, 89.91 \mathrm{C}_{1}, 85.32 \mathrm{C}_{2}, 69.42 \mathrm{C}_{3}, 69.29 \mathrm{C}_{4}, 61.63$ $\mathrm{C}_{5}$. $\left(\mathrm{CH}_{3} \mathrm{COOCH}_{2} \mathrm{CH}_{3}\right.$ : Acetone) (9:1); MS m/z: $362\left(\mathrm{M}^{+}\right.$, 36\%). Anal. Calcd. for $\mathrm{C}_{14} \mathrm{H}_{13} \mathrm{~F}_{3} \mathrm{~N}_{2} \mathrm{O}_{6}$; M.wt: 362.26; C,46.42; H,3.62; F,15.73; N, 7.73; (\%); Found: C, 46.12; H, 3.75; F,15.57; N,7.13 (\%).

\section{Results and Discussion}

The structures of the products 2-8 were established and confirmed on the bases of their elemental analyses and spectral data (IR, ${ }^{1} \mathrm{H}$ and ${ }^{13} \mathrm{C}$ NMR) (see the Experimental section)(Scheme 1). Thus, their ${ }^{1} \mathrm{H}$ NMR spectra of compound 2 showed doublet signals at assigned to the aromatic protons of H-5 H-6 and H-7 and two a singlet signal of amide NH-3 and NH-1.

${ }^{1} \mathrm{H}$ NMR spectra of 5 and $\mathbf{6}$ showed in each case a doublet signals at $\delta 6.48(\mathrm{~d}, 1 \mathrm{H}, J=7.5 \mathrm{~Hz}) \mathrm{H}_{1}$-for compound 5 and at $\delta 6.73(\mathrm{~d}, 1 \mathrm{H}, J=2.2 \mathrm{~Hz}) \mathrm{H}_{1}$ for compound 6 assigned to the anomeric proton of the ribose moiety with spin-spin coupling constant $\left(\mathrm{J}_{1^{\prime}, 2^{\prime}}\right)$ equal to $7.5 \mathrm{~Hz}$, which confirms the $\beta$-anomeric configuration. While confirms the -anomeric configuration showed spin-spin coupling constant $\left(\mathrm{J}_{1^{\prime}, 2^{\prime}}\right)$ equal to $2.2 \mathrm{~Hz}$, which confirms the -anomeric configuration for compound 8 (Break et al, 2014; Break et al, 2013; Break \& Mosselhi, 2012; Mosselhi \& Break, 2011 and Break et al, 2010 and Abdullah Hijazi, 1988). The ${ }^{1} \mathrm{H}$ NMR spectra of nucleosides free showed a doublet signals at $\delta 6.17$ for compound 7 spin- spin coupling constant $\left(\mathrm{J}_{1^{\prime}, 2}\right)$ equal to $7.5 \mathrm{~Hz}$-anomeric configuration and at $\delta 6.53$ for compound 8 assigned to spin-spin coupling constant $\left(\mathrm{J}_{1^{\prime}, 2^{2}}\right)$ equal to $4.5 \mathrm{~Hz}$, which confirms the -anomeric configuration. The ${ }^{1} \mathrm{H}$ NMR of compounds $\mathbf{5}$ and $\mathbf{6}$ showed the expected base moiety protons in addition to the sugar moiety protons (see the Experimental section).

The ${ }^{13} \mathrm{C}$ NMR of nucleoside products revealed the signals are due to the three benzoyl carbonyl groups at and for compound $\mathbf{5}$, and $166.46,165.82$ and 165.40 for compound $\mathbf{6}$, while showed the two signals of amide carbons at 148.17, 146.78 for compound $\mathbf{5}$, and at $147.95 \mathrm{C}_{4}, 147.26 \mathrm{C}_{2}$ for compound $\mathbf{6}$, The twenty one signals at $133.61-128.33$ and at 133.73-128.40 Aromatic carbons for compound 5 and $\mathbf{6}$ respectively.

The five signals were assigned to C-1', C-2', C-3', C-4', and C-5' of the sugar moiety, at $\delta 88.02 \mathrm{C}_{1}, 79.47 \mathrm{C}_{2}, 73.93 \mathrm{C}_{3}$, $70.98 \mathrm{C}_{4}$ and $63.82 \mathrm{C}_{5}$ for compound $\mathbf{5}$, at $\delta 87.46 \mathrm{C}_{1}, 79.37 \mathrm{C}_{2}, 73.90 \mathrm{C}_{3}, 71.69 \mathrm{C}_{4}, 63.83 \mathrm{C}_{5}$, for compound $\mathbf{6}$, at $\delta$ $89.89 \mathrm{C}_{1}, 85.31 \mathrm{C}_{2}, 69.41 \mathrm{C}_{3}, 69.29 \mathrm{C}_{4}, 61.63 \mathrm{C}_{5^{\prime}}$ for compound 7 and at $\delta 89.91 \mathrm{C}_{1}, 85.32 \mathrm{C}_{2}, 69.42 \mathrm{C}_{3}, 69.29 \mathrm{C}_{4}$, $61.63 \mathrm{C}_{5}$ for compound 8 .

The ${ }^{13} \mathrm{C}$ NMR of $\mathrm{CF}_{3}$ group showed at $\delta 119.11,118.01,118.96$ and 118.82 of compounds $(\mathbf{5 , 6 , 7}$ and $\mathbf{8})$ respectively Break and Break. The IR spectrum of compounds $\mathbf{5}$ and $\mathbf{6}$ showed the stretching vibration frequencies of the carbonyl $\mathrm{C}=\mathrm{O}$ groups at $1725 \mathrm{~cm}^{-1}$. IR spectra of compounds $\mathbf{7}$ and $\mathbf{8}$ showed absorptions around $3450 \mathrm{~cm}^{-1}$ for $(\mathrm{OH})$ and 1715 $\mathrm{cm}^{-1}$ for $(\mathrm{C}=\mathrm{O})$.

\section{Conclusion}

Quinazolinone nucleosides are scientific importance in many biologically active compounds. So synthesis and characterization of 8-trifluloromethyl quinazolin-2,4-( $(1 H, 3 H)$-dione $\mathbf{2}$. Ribosylation of compound $\mathbf{3}$ with 1- $O$-acetyl-2,3,5-tri- $O$-benzoyl- $\beta$-D-ribofuranose 4 afforded mixture $\beta$-and $\alpha$-anomeric of the benzoylated nucleoside derivatives 5 and $\mathbf{6}$, respectively. Debenzoylation of the latter affording the corresponding new free N-nucleosides 7 and $\mathbf{8}$, respectively. Nucleosides obtained have been identified by their spectral analysis. 


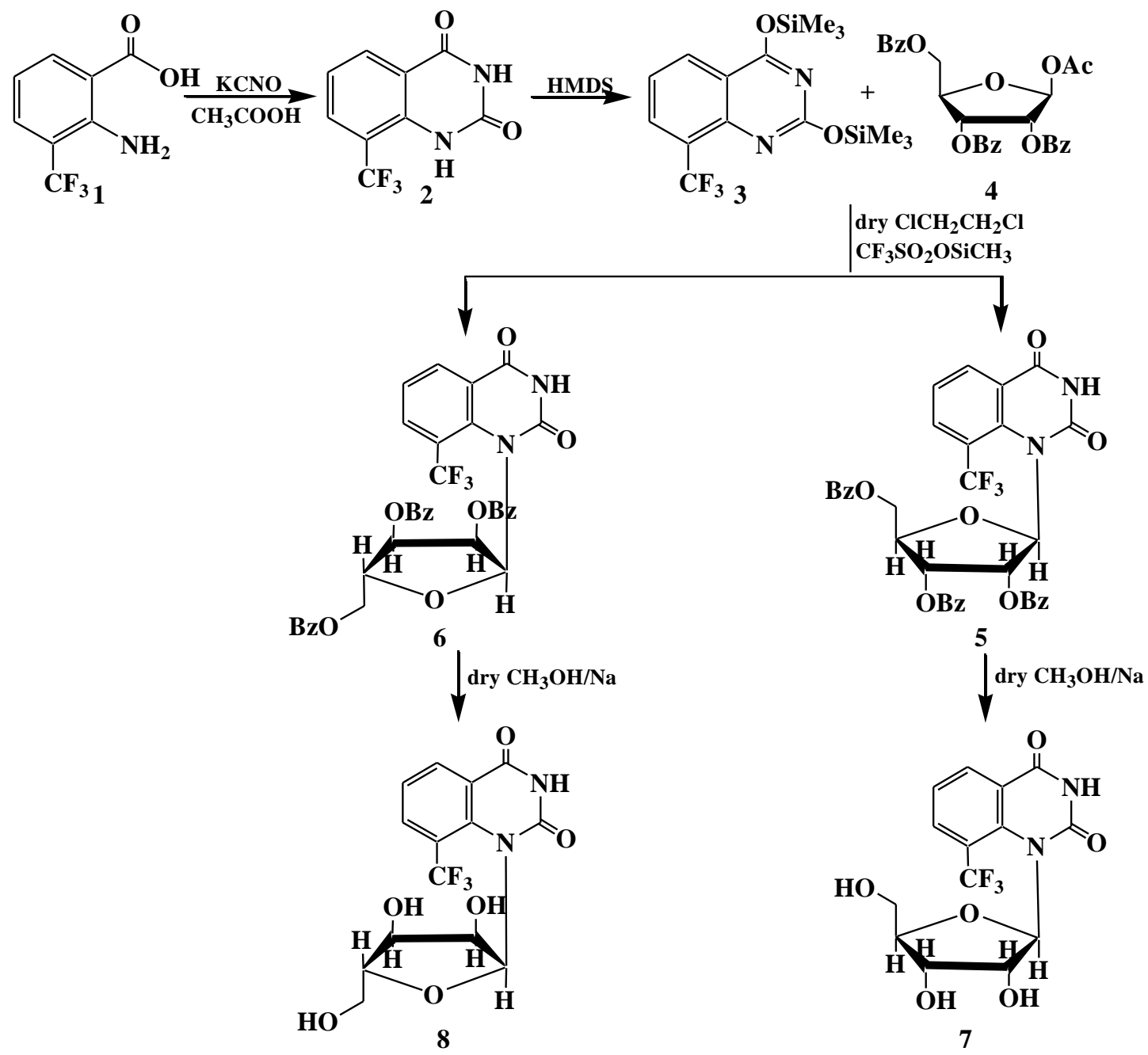

Schem (1). 8-Trifuloro quinazolin-2,4-(3H)-dione Nucleosides

\section{References}

AbdullahM H. (1988). Nucleosides, IV Synthesis and Properties of 2-Metylthio-Naphthimidazole-Ribonucleoside. Nucleosides \& nucleotides, 7(4), 537-547. https://doi.org/10.1080/07328318808075395

Al-Rashood, S. T., Aboldahab, I. A., Nagi, M. N., Abouzeid, L. A., Abdel-Aziz, A. A. M., Abdel-hamide, ... \& El-Subbagh, H. I. (2006). Synthesis, dihydrofolate reductase inhibition, antitumor testing, and molecular modeling study of some new 4(3H)-quinazolinone analogs., Bioorg. Med. Chem., 14, 8608-8621. https://doi.org/10.1016/j.bmc.2006.08.030

Aly, M. M., Mohamed, Y. A., El-Bayouki, K. A. M., Basyouni, W. M., \& Abbas, S. Y. (2010). Synthesis of some new 4(3H)-quinazolinone-2-carboxaldehyde thiosemicarbazones and their metal complexes and a study on their anticonvulsant, analgesic, cytotoxic and antimicrobial activities - Part-1., Eur. J. Med. Chem., 45, 3365-3373. https://doi.org/10.1016/j.ejmech.2010.04.020

Amin, K.M., Kamel, M.M., Anwar, M. M., Khedr, M., \& Syam, Y. M. (2010). Synthesis, biological evaluation and molecular docking of novel series of spiro $[(2 \mathrm{H}, 3 \mathrm{H})$ quinazoline-2,10-cyclohexan]-4(1H)-one derivatives as anti-inflammatory and analgesic agents., Eur. J. Med. Chem., 45, 2117-2131. https://doi.org/10.1016/j.ejmech.2009.12.078

Ayman, M. S., Taha, M. O., Aziz, M. A., Mahmoud A. Al-Qudah, AbuTayehx, R. F. \& Syed A. Rizvi. (2016). Novel anticancer compound [trifluoromethyl-substituted pyrazole N-nucleoside] inhibits FLT3 activity to induce differentiation in acute myeloid leukemia cells, Cancer Letters., 375(2), 199-208. https://doi.org/10.1016/j.canlet.2016.02.028

Break, L. M., \& Mosselhi, A. N. M. (2012). Synthesis, structure and Antimicrobial activity of new 3- and 2- arylmethyl 
and arylacyl-3H [1,2,4] triazino [3,2-b]-quinazoline-2,6 (1H) diones as expect as DNA fluorphores. Research Journal of Chemical Science, 2(5), 23-28.

Break, L. M., Shmiss, N. A. M. M., \& Mosselhi, M. A. N. (2010). Synthesis of some news-nucleoside derivatives of 2-thioxo and (2,4-Dithioxo)-5,6,7,8-Tetrahydrobenzo-Thieno[2,3-d]Pyrimi-din-4-(3H) Ones. Phosphorus, Sulfur and Silicon and the Related Elements, 185(8), 1615-1622. https://doi.org/10.1080/10426500903147159

Chen, H., Chen, W.Q., Gan, L. S., \& Mutlib, A. E. (2003). Metabolism of (S)-5,6-Difluorol-4-Cyclopropylethynyl-4-Trifluorormethyl-3,4-dihydro-2(1H)-Quinazolinone, A non-Nucleoside Reverse Transcriptase Inhibitor, In Human Liver Microsomes. Metabolic Activation and Enzyme Kinetics. Drug Metabolism and Disposition, 31(1), 122-132. https://doi.org/10.1124/dmd.31.1.122

Chien, T. C., Chen, C. S., \& Chern, J. W. (2005). Nucleosides XIII. Facile synthesis of 4-amino-1-(2-deoxy- $\beta$-Dribofuranosyl)quinazolin-2-one as a 2-deoxycytidine analog for oligonucleotide synthesis. Journal of the Chinese Chemical Society, 52(6), 1237-1244. https://doi.org/10.1002/jccs.200500178

Chien, T. C., Chen, C. S., Yu, F. H., \& Chern, J. W.(2004). Nucleosides XI.1) Synthesis and Antiviral Evaluation of 5-Alkylthio-5deoxy Quinazolinone Nucleoside Derivatives as S-Adenosyl-Lhomocysteine Analog, Chem. Pharm. Bull., 52(12) 1422-1426. https://doi.org/10.1248/cpb.52.1422

Diwan, A. R., Robins, R. K., \& Prusoff, W. H. (1969). Antiviral activity of certain substituted purine and pyrimidine nucleosides. Experientia, 25, 98-100. PMID: 4304043. https://doi.org/10.1007/BF01903922

Dunkel, M., \& Pfleiderer, W. (1991). Nucleosides Nucleotides, 10, $799-817$. https://doi.org/10.1080/07328319108046663

Dunkel, M., \& Pfleiderer, W. (1992). Nucleosides Nucleotides, 11, 787-819. https://doi.org/10.1080/07328319208021742

Dunkel, M., \& Pfleiderer, W. (1993). Nucleosides Nucleotides, 12, 125 -374. https://doi.org/10.1080/07328319308021199

Dunkel, M., \& Pfleiderer, W. (1993). Nucleosides. LII. Synthesis and properties of quinazoline-3'-azidonucleosides. Nucleosides and Nucleotides, 12(2), 125-137. https://doi.org/10.1080/07328319308021199

El-Baih, F. E. M., Bakari, S. B. A., \& Hijazi, A. A. (2004). Synthesis and spectroscopic properties of quinazolinedione derivatives. Journal of King Abdelaziz University, 16, 41-53. https://doi.org/10.4197/Sci.16-1.5

Kumar, A., Sharma, P., Kumari, P., \& Kalal, B. L.(2011). Exploration of antimicrobial and antioxidant potential of newly synthesized 2,3-disubstituted quinazoline-4(3H)-ones., Bioorg. Med. Chem. Lett., 21. 4353-4357 https://doi.org/10.1016/j.bmcl.2011.05.031

Kumar, A., Sharma, S., Archana, Bajaj, K., Sharma, S., Panwar, H., ... Srivastava, V. K. (2003). Some new 2,3,6-trisubstituted quinazolinones as potent anti-inflammatory, analgesic and COX-II inhibitors., Bioorg Med Chem, 11, 5293-5299. https://doi.org/10.1016/S0968-0896(03)00501-7

Laila, M. B. (2015). Synthesis of the Novel 3-Benzotriazole-5-yl difluoromethyl-5-trifluoromethyl benzotriazole Nucleosides. International Journal of Chemistry, 7(2), 99. https://doi.org/10.5539/ijc.v7n2p99

Laila, M. B. (2016). Synthesis of Some of Fluorinated Benzimidazole Nucleosides. International Journal of Chemistry. 8(1), 188. https://doi.org/10.5539/ijc.v8n1p188

Laila, M. B., Mohamed, M., \& Abdel-Hafez, S. H. (2014). Synthesis of New Organoselenium Compounds Containing Nucleosides as Antioxidant. Orient. J. Chem., 30(4), 1639-1645. https://doi.org/10.13005/ojc/300423

Laila, M. B., Mosselhi, A. M., \& Elshafai, N. M. (2013). Nucleosides 8 [18]: Ribosylation of Fused Quinazolines-Synthesis of New [1,2,4]Triazolo[5,1-b]- and [1,2,4]Triazino[3,2-b]quinazoline Nucleosides of Fluorescence Interest. Journal of Chemistry. https://doi.org/10.1155/2013/612756

Michel, J., Gueguen, G., Vercauteren, J., \& Moreau, S. (1997). Tetrahedron, 53, 8457-8478. https://doi.org/10.1016/S0040-4020(97)00564-4

Mosselhi, A., Mosselhi, N., \& Laila, M. B. (2011). Nucleosides 79: Synthesis, structure, and biological, activity of new 6-arylidenamino-2-thio- and 2-benzylthiopyrimidine N-nucleosides. Nucleosides, Nucleotides and Nucleic Acids, 30, 681-695. https://doi.org/10.1080/15257770.2011.597628

Mulakayala, N., Kandagatla, B., Ismail, R., Rapolu,K., Rao, P., Mulakayala, C., Kumar, S., Iqbal, J., \& Oruganti, S. (2012). $\mathrm{InCl}_{3}$-catalysed synthesis of 2-aryl quinazolin-4(3H)-ones and 5-aryl pyrazolo[4,3-d]pyrimidin-7(6H)-ones and their evaluation as potential anticancer agents., Bioorg. Med. Chem. Lett., 22, 5063-5066. 
https://doi.org/10.1016/j.bmcl.2012.06.003

Safinaz E. Abbas, Flora, F. B., Hanan, H. G., \& Eman, R. M. (2013). Synthesis and antitumor activity of certain 2,3,6-trisubstituted quinazolin-4(3H)-one derivatives., Bulletin of Faculty of Pharmacy, Cairo University. Volume 51, Issue 2, 273-282. https://doi.org/10.1016/j.bfopcu.2013.08.003

Stout, M. G., \& Robins, R. K. (1968). The synthesis of some quinazoline nucleosides., J. Org. Chem., 33, 1219-1225. https://doi.org/10.1021/jo01267a061

Vittoria, C., Ombretta, L., Daniela, C., Flavia, V., Lucia, S., Chiara, C., ... Stefano, M.(2012). 3-Hydroxy- $1 \mathrm{H}$-quinazoline-2,4-dione derivatives as new antagonists at ionotropic glutamate receptors: Molecular modeling and pharmacological studies. European Journal of Medicinal Chemistry 54, 470-482. https://doi.org/10.1016/j.ejmech.2012.05.036

Vorbruggen, H, Krolikiewicz, K., \& Bennua, B. (1981). Chem. Ber, $114, \quad 1234$. https://doi.org/10.1002/cber.19811140404

Zemplen, G., Gerecs, A., \& Hadacsy, I. (1939). Ber. Dtsch. Chem. Ges., 69, 1827. https://doi.org/10.1002/cber.19360690807

\section{Copyrights}

Copyright for this article is retained by the author(s), with first publication rights granted to the journal.

This is an open-access article distributed under the terms and conditions of the Creative Commons Attribution license (http://creativecommons.org/licenses/by/4.0/). 